\section{Nanoimaging: Methods and Protocols}

\author{
A.A. Sousa and M.J. Kruhlak, eds.
}

New York, NY: Humana Press-Springer, 2013, 510 pages, $\$ 159$

A common goal in biology is to dissect the astonishing complexity of the cellular molecular machinery, seeking to understand how individual components of this machinery assemble to form higher-order structures, navigate the cell with synchronized dynamics, and interact to produce a given biologic outcome. Imaging has been an integral tool in biology, allowing researchers to view the detailed molecular biology responsible for coordinating cellular life. Today, advanced imaging modalities enable the visualization of biologic specimens at a range of length scales and resolutions, in multiple dimensions (both time and space), with chemical or molecular specificity, and from the surface or bulk of the specimen, revealing the secrets of the molecular bionetwork within the cell.

The book is divided into 4 parts, each containing several chapters pertaining to a given field. Part I, with 9 chapters, covers light microscopy techniques with a special emphasis on imaging at spatial resolutions that go beyond the important Abbe (diffraction) limit. Part II, with 7 chapters, contains several established and powerful techniques in electron microscopy for high-resolution imaging of molecules, cells, and tissues, in both 2 and 3 dimensions. Part III, with 4 chapters, deals with the unique methods that reveal the advantages of scanning probe microscopy for imaging and probing macromolecular complexes and membrane surface topography. Part IV, with 5 chapters, closes the book with correlative microscopy techniques, soft x-ray tomography, and secondary ion mass spectrometry imaging. Keeping up with the tradition of the Methods in Molecular Biology series, chapters are largely composed of detailed protocols describing a specific experimental procedure. Figures are mostly clear, and the tables and index are helpful.

This book is a timely volume and achieves its mission of highlighting many of the most exciting possibilities in microscopy for the investigation of biologic structures at nanolength molecular scales. I highly recommend this book to academic radiologists, imaging scientists, and molecular biologists.

\section{E. Edmund Kim}

University of California at Irvine

101 The City Dr. S.

Orange, CA 92868

E-mail: edmundek@uci.edu

Published online Mar. 27, 2013.

DOI: 10.2967/jnumed.113.121624

COPYRIGHT @ 2013 by the Society of Nuclear Medicine and Molecular Imaging, Inc.

\section{Nanoimaging}

B.A. Goins and W.T. Phillips, Eds.

Singapore: Pan Stanford Publishing, 2011, 298 pages, $\$ 149.95$

Nanocarriers have been used extensively to effectively deliver drug molecules to cancer tissue and cells and to tumor vessels. Nanocarriers also can incorporate different kinds of agents such as drugs, imaging probes, and specific ligands. There have been significant advances in the fabrication of new nanostructures, including nanoparticles, as well as new imaging equipment and contrast agents.

This 13-chapter book is part of the Pan Stanford Series on Biomedical Nanotechnology and devoted especially to reviewing the current status of the use of nanoparticles in imaging applications in vivo. Chapter I deals with nanoparticles for oncologic imaging. Chapters II-V focus on in vivo imaging of iron oxide particles and liposomes. Chapters VI-XII highlight the use of new nanoparticles that are currently at the preclinical stage of testing, such as quantum dots, dendrimers, polymer-based nanoparticles, gold nanoshells, carbon nanotubes, and metal nanorods. The final chapter, XIII, discusses microbubbles-ultrasound contrast agents with a diameter on the order of micrometers.

This book has attempted to represent the major clinical imaging modalities such as MR imaging, CT, PET, SPECT, ultrasonography, and optical imaging. The book also highlights the specific advantages of nanoparticles for drug delivery and imaging applications. The versatile nature of nanoparticles allows for their use as multimodality imaging agents as well as theranostics for carrying both diagnostic and therapeutic agents. The figures are clear and useful. Readers will find that principles learned from the in vivo imaging of one type of nanoparticle are useful for investigations of other types of nanoparticles. I highly recommend this book to imaging scientists, molecular biologists, academic radiologists, and oncologists.

\section{E. Edmund Kim}

University of California at Irvine

101 The City Dr. S.

Orange, $C A 92868$

E-mail:edmundek@uci.edu

Published online Mar. 26, 2013.

DOI: 10.2967/jnumed.113.121632 